Table 1 Midtimes* of uranian ring occultations

\begin{tabular}{ccc}
\hline Ring & Immersion & Emersion \\
6 & $19: 18: 56.56$ & $20: 17: 50.43$ \\
5 & $19: 18: 31.03$ & $20: 18: 11.02$ \\
4 & $19: 18: 19.24$ & $20: 18: 27.74$ \\
$\alpha$ & $19: 16: 30.51$ & $20: 20: 18.76$ \\
$\beta$ & $19: 15: 42.38$ & $20: 21: 05.74$ \\
$\eta^{\dagger}$ & $19: 14: 25.46$ & $20: 22: 21.60$ \\
$\gamma$ & $19: 14: 02.63$ & $20: 22: 44.16$ \\
$\delta$ & $19: 13: 29.49$ & $20: 23: 18.05$ \\
$\varepsilon$ & $19: 11: 10.02$ & $20: 25: 28.10$ \\
\hline
\end{tabular}

* On 26 April 1981 uT.

$\uparrow$ The occultation times for the $\eta$ ring refer to the narrow core at its inner edge ${ }^{9}$.

of our model is still significantly larger than the error in determining a ring occultation time. Hence, further improvements to our model should result in even more information about the Uranus system.

We thank A. Freedman, S. Tremaine and P. Goldreich for helpful discussions. This work was supported in part by NSF grant AST-8010699 and NASA grant NSG 7526.

Received 9 June: 3ccepted 25 June 1982.

1. Smoluchowski, R. Nature 280, 377-378 (1979)

2. Elliot, J. L et al. Nature $294,526-529$ (1981).

3. Burns, J. A. in The New Solar System, 129-142 (Sky Publishing, Cambridge, Massachusetts, 1981).

4. Elliot, J. L., Dunham, E., Wasserman, L. H., Millis, R. L. \& Churms, J. Astr. J. 83, 980-992 (1978)

5. Nicholson, P. D., Persson, S. E., Matthews, K., Goldreich, P. \& Neugebauer, G. Astr. J. 83, 1240-1248 (1978)

6. Elliot, J. L. et al. Astr. J. 86, 127-134 (1981).

7. Nicholson, P. D., Matthews, K. \& Goldreich, P. Astr. J. 86, 596-606 (1981)

8. Nicholson, P. D. Matthews, K. \& Goldreich, P. Astr. J. 87, 433-447 (1982)

9. Elliot, J. L. et at. Astr. J. 86, 444-445 (1981)

10. French, R. G. et al. Icarus (submitted).

11. Goldreich, P. \& Tremaine, S. Astr. J. 84, 1638-1641 (1979)

12. Smoluchowski, R. Commun. Astrophys. 8, 69-78 (1979).

3. Aggarwal, H. R. \& Obserbeck, V. R. Astrophys. J. 191, 577-588 (1974).

4. Goldreich, P. \& Tremaine, S. Nature 277, 97-98 (1979).

15. Goldreich, P. \& Tremaine, S. Astrophys. J. 243, 1062-1075 (1981)

\section{An example of induced centrifugal force in general relativity}

\author{
Jeffrey M. Cohen*, William J. Sarill $\dagger$ \\ \& C. V. Vishveshwara \\ * Physics Department, University of Pennsylvania, Philadelphia, \\ Pennsylvania 19104, USA \\ † Physics Department, Boston University, Boston, \\ Massachusetts 02215, USA \\ ¥ Raman Research Institute, Bangalore 560 080, India
}

Thirring's solution ${ }^{1}$ for a rotating spherical mass is cited often as an example which exhibits induced Coriolis and centrifugal forces in general relativity ${ }^{2,3}$. Previously, it was pointed out $\mathbf{t}^{4}$ that the 'centrifugal terms' proportional to the square of the angular velocity actually represented quadrupole effects. These effects arose as a consequence of the latitude-dependent velocity distribution which generated an axially symmetric (nonspherical) mass distribution. This raises the question: Are there solutions to Einstein's equations which exhibit induced centrifugal force? If no such solutions exist, then the concept of induced rotation of inertial frames breaks down. Such a lack of connection between rotating masses and induced inertial frame rotation would in turn imply a breakdown of the
Machian $^{5,6}$ idea that an inertial frame is a system of reference in which the stress-energy of the universe is at rest on the average $^{7}$. Here we demonstrate that there are solutions to Einstein's equations which exhibit induced centrifugal force (other solutions may also be of interest in this connection ${ }^{\mathbf{8}}$ ).

As pointed out elsewhere ${ }^{2}$, velocity-dependent density distributions can give terms easily misinterpretable as centrifugal force-a difficulty which can be circumvented by treating a body for which the symmetry of the density distribution is velocity-independent; such a body is a rotating cylindrical shell. Other important advantages of this choice of source geometry are that it generates a simple geometry within the shell, that is, the flat space-time (ref. 9 and W.J.S., unpublished thesis), and the solution is exact. When written in the appropriate coordinates, flat space is well understood and easily interpreted. Furthermore, as the solution is exact, no ambiguities arise due to approximations.

A rotating cylindrical shell can be treated using a generalization of the Weyl-Levi-Civita ${ }^{10,11}$ metric

$$
\mathrm{d} s^{2}=-\mathrm{e}^{+2 \psi} \mathrm{d} t^{2}+\mathrm{e}^{2(\gamma-\psi)}\left(\mathrm{d} r^{2}+\mathrm{d} z^{2}\right)+r^{2} \mathrm{e}^{-2 \psi}(\mathrm{d} \phi-\Omega \mathrm{d} t)^{2}
$$

and the corresponding vacuum field equations

$$
\begin{gathered}
\nabla^{2} \psi=\frac{1}{2} r^{2} \mathrm{e}^{-4 \psi} \nabla \Omega \cdot \nabla \Omega \\
\nabla \cdot\left(r^{2} \mathrm{e}^{-4 \psi} \nabla \Omega\right)=0
\end{gathered}
$$

derived by Ehlers ${ }^{12}$ and later independently by the authors (ref. 13 and J.M.C. and W.J.S., unpublished). Here $\nabla$ and $\nabla^{2}$ are the usual flat space operators. The function $\gamma$ is determined by quadrature from the equation

$$
\gamma_{r}=r \psi_{r}^{2}-\frac{1}{4} r^{3} \mathrm{e}^{-4 \psi} \Omega_{r}^{2}
$$

where the subscript denotes differentiation with respect to $r$ and cylindrical symmetry has been assumed so that the derivatives with respect to $z$ vanish. Inside the shell of uniform density, the only non-singular stationary exact solution is the flat space metric in rotating coordinates

$$
\mathrm{d} s^{2}=-\mathrm{d} t^{2}+\mathrm{d} r^{2}+\mathrm{d} z^{2}+r^{2}(\mathrm{~d} \phi-\Omega \mathrm{d} t)^{2}
$$

where $\Omega$ is a constant. This rotating form of the flat space-time is used to obtain an explicitly smooth match across the rotating shell; this form also facilitates an unambiguous identification of the Coriolis and centrifugal terms. The metric outside the shell can be obtained from the Weyl-Levi-Civita metric via the multivalued transformation $\mathrm{d} t^{\prime}=\mathrm{d} t+K \mathrm{~d} \phi$ where $K$ is a constant. The matching across the shell relates the constants $\Omega$ and $K$ to the mass per unit length and angular velocity $\omega$ of the shell.

It is generally known that the geodesic equations corresponding to the flat space-time metric (3) contain Coriolis terms proportional to $\Omega$ and centrifugal terms proportional to $\Omega^{2}$. The frame in which all these terms vanish simultaneously is defined as the inertial frame. When the angular velocity $\omega$ of the shell vanishes, $\Omega$ also vanishes. Thus, $\Omega$ can be interpreted as the induced angular velocity associated with the dragging of inertial frames. We conclude, therefore, that there are solutions of Einstein's equations which exhibit induced centrifugal as well as Coriolis forces in accordance with Machian ideas.

This work was supported in part by the NSF.

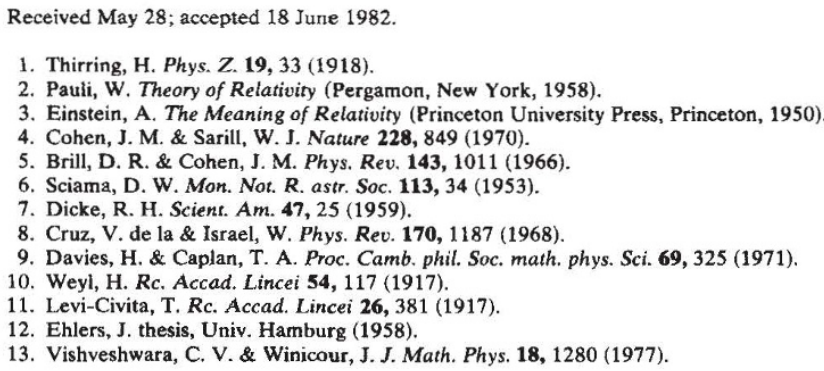

